\section{HEALTH AND SAFETY}

\section{Inside and outside}

Environ. Sci. Technol. 42, 4600-4606 (2008)

As the production of nanomaterials increases it becomes ever more important to know the exposure levels experienced by workers during the manufacturing process. Researchers from Virginia Tech measured airborne particles in a small commercial facility producing fullerenes and other carbonaceous nanomaterials, and found them to be well contained.

Linsey Marr and colleagues measured the concentrations of airborne particles at three different locations inside a production facility over a period of time. The fine-particle mass concentrations, submicrometre size distribution and the carbonaceous content of the particles were measured inside the fume hood where the materials were produced, just outside the fume hood where the technicians worked, and in the background. Physical handling of the materials and production activities, such as opening the reactor, increased the number of aerosolized particles, but the fume hood was effective at containing them.

The average mass concentration and particle numbers inside the facility were not significantly different from outside, but daily and hourly variations of ambient particles may affect the measurements, and this is one of several areas in which Marr and co-workers call for further research.

\section{GRAPHENE NANORIBBONS}

\section{Quantum conductors}

Preprint at http://arxiv.org/abs/0805.0035 (2008)

Graphene has great potential for carbon-based electronics owing to its unusual electronic transport properties. It has been shown that graphene 'nanoribbons' can effectively confine charge carriers to move in one dimension, which is similar to what happens in a carbon nanotube. Ribbons with the correct width and crystal structure are expected to have useful semiconducting properties and may also display quantum behaviour on a relatively large scale. Yu-Ming Lin and co-workers at the IBM TJ Watson Research Center have now provided experimental evidence that electrical conductance can be quantized inside graphene nanoribbons.
The IBM team cut sheets of graphene into ribbons just $30 \mathrm{~nm}$ wide and measured the conductance as the voltage difference between the two ends of the nanoribbon was increased. At room temperature, the conductance increased smoothly with voltage. However, at $80 \mathrm{~K}$ the conductance reached plateaus at certain voltages before increasing again - a sign that the conductance is quantized. The plateaus and steps are caused by the electrons and holes in the ribbon having to overcome energy gaps that they cannot travel around because of the narrowness of the ribbon.

\section{MOLECULAR ELECTRONICS Up the junction}

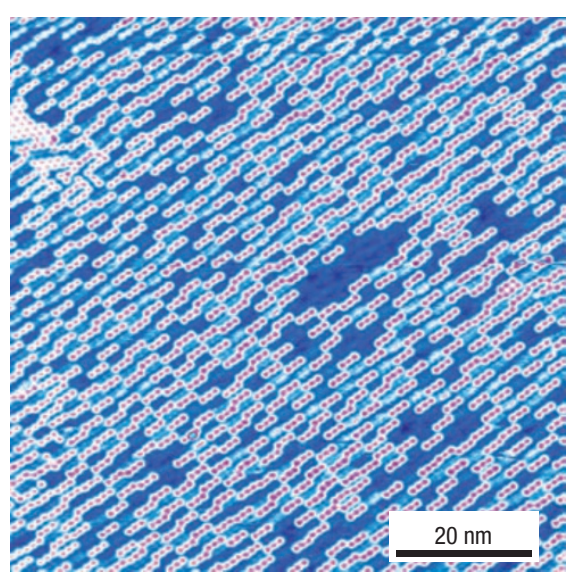

\section{Appl. Phys. Lett. 92, 193301 (2008)}

The dimensions of organic molecules offer a direct 'bottom-up' means of producing functional components for nanoelectronic circuits. However, despite extensive research, it is still difficult to make selfassembled molecular structures with useful functions such as rectifying junctions. Now, Andrew Wee of the National University of Singapore and co-workers have made self-assembled nanojunction arrays by evaporating carbon-60 molecules onto p-sexiphenyl (6P) nanostripes on silver substrates. The carbon- 60 is the electron acceptor, and the $6 \mathrm{P}$ is the donor.

The 6P nanostripes had widths of $2.95 \mathrm{~nm}$ and a periodicity of $\sim 0.7 \mathrm{~nm}$. When half a monolayer of carbon- 60 was deposited and then annealed for 30 minutes at $350 \mathrm{~K}$, the result was a twodimensional array of carbon-60 'triplets' located on top of the $6 \mathrm{P}$ chains. Annealing for a further 30 minutes at $380 \mathrm{~K}$ led to the insertion of linear chains of carbon- 60 molecules between the 6P nanostripes. The ability to produce both vertical and lateral donor/acceptor nanojunctions with organic molecules could lead to improved solar cells.

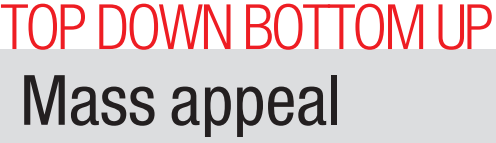

Nanoparticles can improve the spatial resolution of mass spectrometry imaging of mammalian tissues.

Back in his assistant professor days in the 1990s, Mitsutoshi Setou was looking for a company to fund and commercialize his ideas. Setou, who is now a full professor at the Mitsubishi Kagaku Institute of Life Sciences in Tokyo, wanted to exploit nanoparticles to improve the resolution of mass spectrometry imaging (MSI) - an analytical technique that is used to visualize the spatial distribution of compounds. Only one person took the proposal seriously, but luckily for Setou that person was Shigehiko Hattori, the president of the Japanese equipment company Shimadzu. Part of the problem might have been Setou's relative youth he was 33 at the time, whereas most team leaders in Japan tend to be 55 or older.

Ionizing samples is a central part of MSI, and Setou - working with Yuko Ichiyanagi, a physicist at Yokohama National University, and others - has recently developed silicon dioxide-based nanoparticles with hydrophilic groups on their surface to assist with this process when studying mammalian tissue specimens. Traditional reagents based on organic solutions form large crystals when sprayed on the specimens and this decreases the imaging resolution. Setou's nanoparticles - which have a diameter of just $3.7 \mathrm{~nm}$ across - do not suffer from this problem (Anal. Chem. 80, 4761-4766; 2008).

"Letting biologists, physicists and chemists understand nanotechnologybased MSI is a possible, meaningful and fruitful mission," says Setou. "Everyone was sceptical at the beginning and changing everyone's way of thinking was most difficult but also most rewarding." What advice does he have for others facing similar challenges? "We need to study a lot," he says, "and we should not hesitate to ask naïve questions to understand the different sciences because we do not have to possess all the skills in every technology."

The definitive versions of these Research Highlights first appeared on the Nature Nanotechnology website, along with other articles that will not appear in print. If citing these articles, please refer to the web version. 